\title{
Tolerance to Sound Intensity of Binaural Coincidence Detection in the Nucleus Laminaris of the Owl
}

\author{
Jose Luis Peña, Svenja Viete, Yehuda Albeck, and Masakazu Konishi \\ Division of Biology, California Institute of Technology, Pasadena, California 91125
}

Neurons of the owl's nucleus laminaris serve as coincidence detectors for measurement of interaural time difference. The discharge rate of nucleus laminaris neurons for both monaural and binaural stimulation increased with sound intensity until they reached an asymptote. Intense sounds affected neither the ratio between binaural and monaural responses nor the interaural time difference for which nucleus laminaris neurons were selective. Theoretical analysis showed that high afferent discharge rates cause coincidence detectors with only excitatory input to lose their selectivity for interaural time difference when coincidence of impulses from the same side becomes as likely as that of impulses from the two sides. We hypothesize that inhibitory input whose strength increases with sound intensity protects nucleus laminaris neurons from losing their sensitivity to interaural time difference with intense sounds.

Key words: acoustic localization; interaural time difference; sound intensity; coincidence detection; nucleus laminaris; owl
Coincidence detection is an important operation in many neural functions such as computation of interaural time difference (Jeffress, 1948), direction of visual movement (Reichardt, 1961), echo delays (Suga et al., 1990), and pattern recognition (Hopfield, 1995). Most coincidence detector models receive afferent impulses by one axon in each of the two input channels. If multiple axons deliver afferent impulses in each channel, these impulses can arrive at the detector simultaneously. This condition may pose problems in some systems. High afferent impulse rates in such a system increase the chance of same-side coincidence that may not be distinguishable from coincidence between the two input channels. Models of binaural coincidence detection also show that detectors with only excitatory input saturate for high afferent impulse rates (Albeck, 1994; Reed and Durbeck, 1995). Neurons of the barn owl's nucleus laminaris (NL) serve as coincidence detectors for measurement of interaural time difference (ITD) (Sullivan and Konishi, 1984; Carr and Konishi, 1988, 1990). NL receives bilateral input from magnocellular nuclei (NM) (Takahashi and Konishi, 1988a). Carr and Boudreau (1993) estimated that $45-150 \mathrm{NM}$ axons from each side innervate a single NL neuron. In this paper, we study the effect of sound intensity on ITD sensitivity and present evidence and theory suggesting that inhibition prevents NL neurons from losing their sensitivity to interaural time difference (ITD) with intense sounds.

\section{MATERIALS AND METHODS}

Parameters used in the model

$a_{0}(\mathrm{NA})=a_{0}(\mathrm{NM}) / 2$ : effect of intensity on NM/NA

$a_{1}(\mathrm{NM})=2.7$ : controls phase locking in $\mathrm{NM}$

Received June 10, 1996; revised Aug. 7, 1996; accepted Aug. 9, 1996.

This work was supported by National Institute of Neurological Disorders and Stroke Grant DC-00134 and postdoctoral fellowships from the Pew Latin American Fellows Program (J.L.P.), the Deutsche Forschungsgemeinschaft (S.V.), and the Sloan Center for Theoretical Neurobiology at Caltech (Y.A.). We thank Jamie Mazer and Chris Malek for assistance with computer programming and Catherine Carr, Roian Egnor, Jamie Mazer, Terry Takahashi, Larry Proctor, and Marc Schmidt for reading an early version of this paper. Ben Arthur commented on this manuscript. Correspondence should be addressed to Masakazu Konishi, Division of Biology 216-76, California Institute of Technology, Pasadena, CA 91125.

Copyright (C) 1996 Society for Neuroscience $0270-6474 / 96 / 167046-09 \$ 05.00 / 0$
$a_{1}(\mathrm{NA})=0.0$ : no phase locking in NA

refractory $(\mathrm{NL})=1 \mathrm{msec}$ : refractory period for NA, NM, NL

$\tau_{\mathrm{ex}}=1 \mathrm{msec}$ : decay time constant of excitatory "current"

$\tau_{\text {in }}=2$ msec: decay time constant of inhibitory "current"

$T(\mathrm{NL})=3.0$ : threshold current for firing

$w_{\mathrm{ex}}=1.0:$ excitatory weight

$w_{\text {in }}=0.2$ : inhibitory weight

\section{Surgery}

Data were obtained from 11 adult barn owls (Tyto alba) of both sexes. These animals also provided data for other studies of the nucleus laminaris. The owls were anesthetized by intramuscular injection of ketamine hydrochloride $(25 \mathrm{mg} / \mathrm{kg}$; Ketaset) and diazepam $(1.3 \mathrm{mg} / \mathrm{kg}$; Western Medical Supply). An adequate level of anesthesia was maintained with supplemental injections of ketamine when necessary. Body temperature was maintained with a heating pad. The skull was immobilized by placing the owl in a stereotaxic head holder such that the palatine ridge (the roof of the upper mandible) was inclined down by $70^{\circ}$ with respect to the horizontal bars of the stereotaxic apparatus using the ear bars as the pivotal point. A small stainless steel plate cemented to the skull held the head at this angle, and a reference point was glued onto the skull at the intersection of the interaural axis and the midline of the skull.

Recording sessions began 5-7 d after the implantation of the head plate. Anesthesia was induced and maintained as described above. A small craniotomy was made overlying NL. The dura mater was incised for electrode insertion. Recording sessions lasted several hours, after which the craniotomy was covered with a plastic sheet the edges of which were sealed on the skull with a thin layer of dental cement, and the skin incision was closed and treated with antibiotic ointment (bacitracin zincneomycin sulfate-polymyxin B sulfate; E. Fougera \& Co.). The owls were returned to their individual cages and monitored for their recovery. Depending on the owls' weight and recovery, experiments were repeated every $7-10 \mathrm{~d}$ for a period of several weeks.

\section{Acoustic stimuli}

Sound stimulus synthesis, data collection, and analysis were carried out with custom software (Mazer, 1995). All experiments were performed in a double-walled sound-attenuating chamber. Acoustic stimuli were delivered by earphones (Sony MDR-E535) attached to a metal delivery tube (3 $\mathrm{cm}$ long, $4 \mathrm{~mm}$ inner diameter). A small microphone (ED-1939, Knowles Electronics, Itasca, IL) with a probe tube was used to measure sound intensity at the end of the delivery tube. The gaps between the earphone assembly and the ear canal were filled with silicone impression material (Gold Velvet, JKR Laboratories, Wichita, KS). Sound intensities were measured in the ear canal at a distance of $\sim 1 \mathrm{~mm}$ from the ear 
drum using a $12.5 \mathrm{~mm}$ B \& $\mathrm{K}$ microphone with a calibrated probe tube (1 $\mathrm{mm}$ outer diameter, $5 \mathrm{~mm}$ length). This tube was inserted through a hole made in the squamosal bone that forms the roof of a cavity over the ear drum. Simultaneous measurement of sound with both the B \& K and the Knowles microphones made it possible to translate the voltage output of the Knowles into sound intensity in $\mathrm{dB}$ sound pressure level (SPL). The Knowles microphones were then used to calibrate the earphone assemblies at the beginning of each experimental session.

The calibration data contained the amplitudes and phase angles measured in steps of $100 \mathrm{~Hz}$. Differences in amplitude between the two earphones could usually be reduced by repositioning the earphones. For phase differences, appropriate corrections were made in the affected data. Irregularities in the frequency response of each earphone were automatically smoothed by the computer from 4 to $9 \mathrm{kHz}$. The study of neuronal responses was restricted to cells tuned to frequencies above $4 \mathrm{kHz}$, because lower frequency sounds pass from one middle ear to the other through the interaural canal (Moiseff and Konishi, 1981). This cross-talk can confound the study of neurons tuned to lower frequencies.

Acoustic stimuli were synthesized on a computer (Sparc/IPX, Sun Microsystems) and presented by a digital signal processor equipped with a 16 bit, $48 \mathrm{kHz}$ data acquisition subsystem (S56x + Proport, Berkeley Camera Engineering). Tonal and broadband stimuli $100 \mathrm{msec}$ in duration, 5 msec linear rise/fall time, were presented once per second. ITD was varied in steps of either one-tenth of the period for tonal stimuli or $30 \mu \mathrm{sec}$ for noise stimuli. Stimulus intensities could be varied in steps of $1 \mathrm{~dB}$ with a pair of digitally controlled attenuators (PA4, Tucker Davis Technologies).

We varied sound intensity equally in the two ears in most of the experiments in this paper. The term average binaural intensity (ABI) refers to the sum of sound intensities in the two ears divided by 2 . Thus, for example, if sound intensity is $40 \mathrm{~dB}$ SPL in one ear and $60 \mathrm{~dB}$ SPL in the other, the ABI is $(40+60) / 2=50 \mathrm{~dB}$ SPL.

\section{Data collection}

All data were obtained with a "loose patch" technique, which permitted well isolated and stable extracellular recordings (Fig. 1). This is an important technical advance in the study of NL, because isolation of single neurons is very difficult to obtain, presumably because of the sparsely distributed neuronal somata and the large field potentials present in this area. Even if neurons are isolated, they are difficult to maintain mostly because of brain pulsations. Similar difficulties have been encountered in most of the studies in both NL and medial superior olivary nucleus (MSO) of mammals (Moushegian et al., 1964, 1967, 1975; Rupert et al., 1966; Goldberg and Brown, 1969; Guinan et al., 1972; Crow et al., 1978; Caird and Klinke, 1983; Moiseff and Konishi, 1983; Sullivan and Konishi, 1984; Carr and Konishi, 1990; Yin and Chan, 1990). In the present study, the number of neurons that could be obtained in each experimental session was still small, but the neurons could be maintained for 1-2 hr during which an extensive test protocol could be carried out.

Patch electrodes were prepared from $1.0 \mathrm{~mm}$ borosilicate glass (World Precision Instruments) using a micropipette puller (Sutter Instruments $\mathrm{P}$-87). Electrodes were filled with a patch solution (in mM: K-gluconate 100, EGTA 10, HEPES 40, $\mathrm{MgCl}_{2}$ 5, Na-ATP 2.2, Na-GTP 0.3). Electrode impedance ranged from 4 to $10 \mathrm{M} \Omega$. Broad-band noise bursts with ITD and IID set to zero were used as search stimuli. NL was located stereotaxically and by its physiological response properties. At $1.5-2.5$ $\mathrm{mm}$ posterior to the interaural axis and $1.5-2.0 \mathrm{~mm}$ from the midline, NL is usually $8-19 \mathrm{~mm}$ below the surface of the brain. NL can also be recognized by neurophonic potentials that closely resemble the stimulus waveform. In the owl's brainstem, NM and NL are the only nuclei that produce neurophonic potentials, presumably because their neurons phase-lock to the stimulus. NL neurophonic potentials show ITD tuning, whereas those in NM do not.

Electrodes were advanced with a microdrive (Motion Controller, Model PMC 100, Newport) in steps of $100 \mu \mathrm{m}$ until NL was reached. The size of the steps was reduced to 3-5 $\mu \mathrm{m}$ to search for and isolate single neurons in NL. During this process, a positive pressure was applied to the tip of the electrode to prevent clogging, which could be detected easily by changes in impedance. When small sound-evoked impulses became recognizable, we carried out a simple test for discriminating between monaural and binaural responses. Application of a negative pressure at the tip of the electrode often led to good isolation of the spiking cell even without achieving a high-resistance seal. Once this degree of isolation was obtained, the electrode was seldom dislodged from the cell. We have been able to maintain cells over $2 \mathrm{hr}$ without any sign of diminishing
A \# 030195-396.02.02

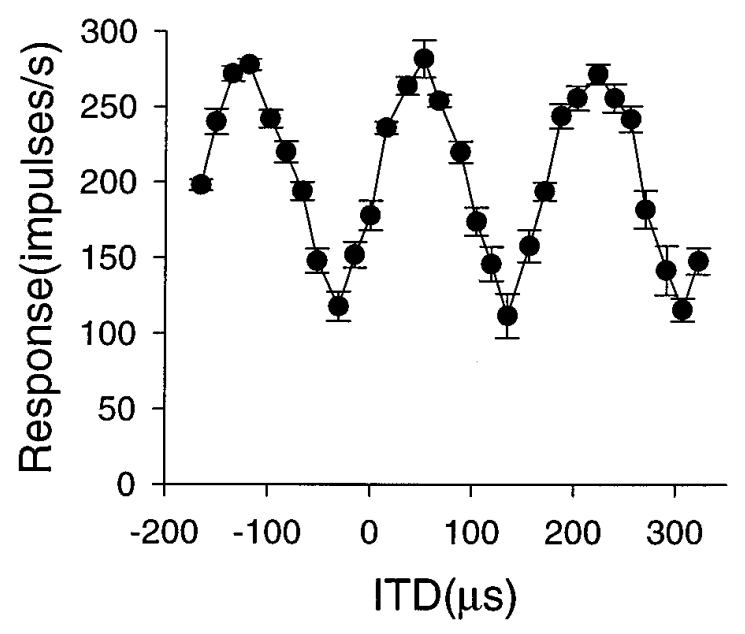

B


C
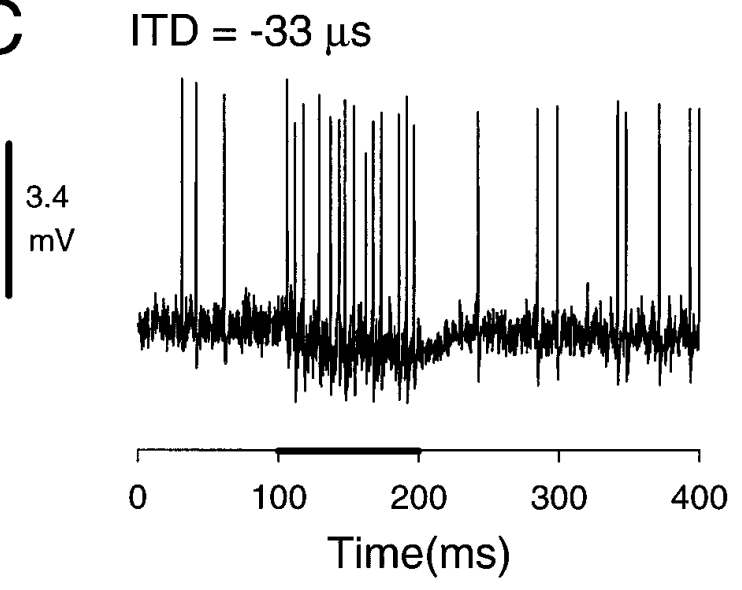

Figure 1. Clean isolation and stable recording of NL neurons. A, ITD tuning curve of an NL neuron (best frequency $5900 \mathrm{~Hz}$ ). Number in the top left corner is neuron ID number; error bars represent SEM. $B$, Single trace of the neuron's response to the most favorable ITD $(-118 \mu \mathrm{sec})$. $C$, Single trace of the neuron's response to the least favorable ITD $(-33 \mu \mathrm{sec})$. The bar on abscissa indicates the duration of the tone burst.

impulse amplitude. The health of the cells gradually deteriorates in conventional whole-cell patch recording, because they lose ions and other molecules by diffusion into the electrode. This problem did not occur with our method, because the electrode did not appear to break the cell membrane; it worked as a suction electrode. Neural signals were recorded with an Axoclamp-2A amplifier (Axon Instruments, Foster City, CA) in 
Figure 2. Methods of determining threshold, asymptote, and dynamic range. An example showing how these parameters were estimated. See text for details.

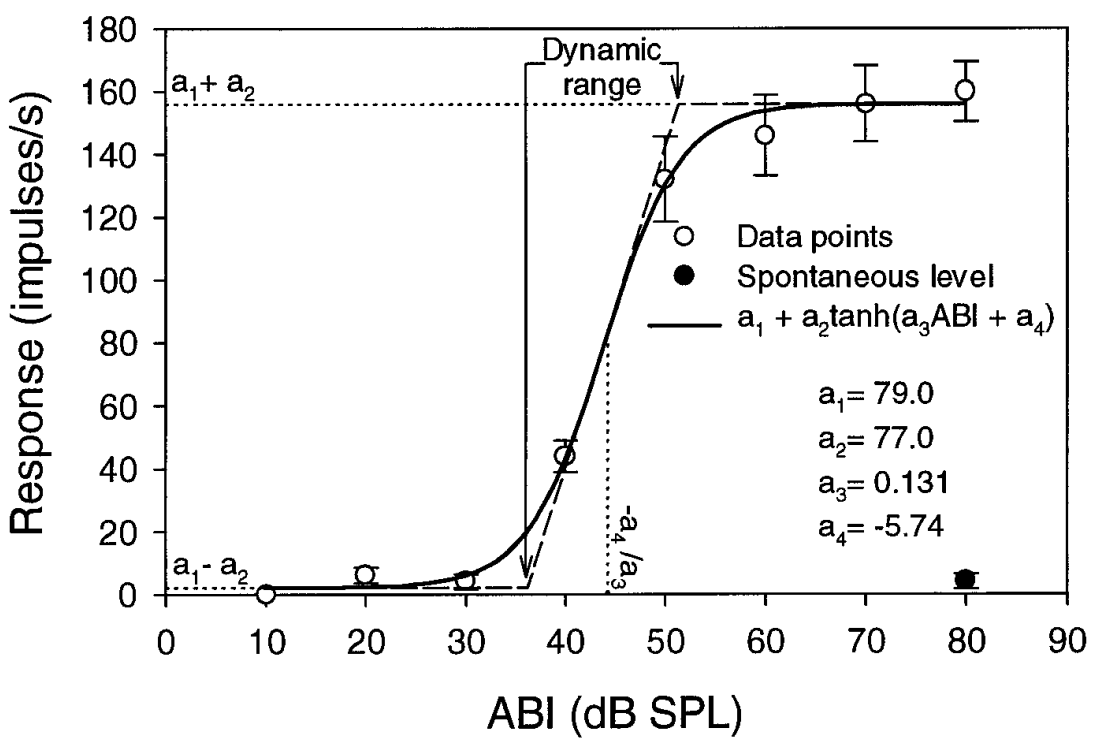

(MIP = mean interaural phase), which is determined by the following equation:

$$
\begin{aligned}
\mathrm{MIP} & =\arctan \left(\sum y_{\mathrm{i}} / \sum x_{\mathrm{i}}\right) \\
x_{\mathrm{i}} & =R_{\mathrm{i}} \cos \beta \\
y_{\mathrm{i}} & =R_{\mathrm{i}} \sin \beta .
\end{aligned}
$$
after the stimulus offset. Spontaneous activity was measured by counting the number of impulses occurring in the same time window for "spontaneous trials" which were randomly interleaved with stimulus trials. Once a well isolated and stable NL recording was obtained, the cell was studied according to a protocol that included rate-intensity curves for monaural stimulation, isointensity frequency tuning curves, and ITD curves for different average binaural intensities (ABIs).

The same loose patch method was used to record from NM axons. Axons coming from both the ipsilateral and the contralateral NM could be easily recorded as they course across NL (cf. Carr and Konishi, 1990). The data from these recordings were used for comparison with NL neurons.

\section{Data analysis}

Threshold, asymptote, and dynamic range. Rate-intensity curves were constructed from data in which impulses were recorded for different sound levels. Because determination of thresholds, asymptotic levels, and dynamic ranges by eye is subjective, an objective and automatic method was developed. Impulse rate-intensity curves were approximated by sigmoidal curves (Fig. 2). Thus, the impulse rate as a function of sound intensity was fitted to:

$$
\text { Response }(\mathrm{ABI})=a_{1}+a_{2} \tanh \left(a_{3} \mathrm{ABI}+a_{4}\right)
$$

The fit used the Levenberg-Marquardt technique. Initial values for the fitting algorithm were automatically estimated from the data. From the four fit parameters, three quantities were computed. The minimal or spontaneous firing level was $a_{1}-a_{2}$. The maximal or saturation level was $a_{1}+a_{2}$. The maximal slope of the response was $a_{2} a_{3}$. Then a straight line was drawn having a slope of $a_{2} a_{3}$ and passing the point where the $\mathrm{ABI}=$ $-a_{4} / a_{3}$, corresponding to the midpoint between the minimum and the maximum. The dynamic range of the neuron was defined as the interval on the ABI axis between the value where this line intersects the minimal level and the value where the line intersects the maximal level. Thus, the estimated dynamic range starts at $-\left(a_{4}+1\right) / a_{3}$ and ends at $-\left(a_{4}-1\right) / a_{3}$ (Fig. 2).

Mean interaural phase and vector strength. The periodic properties of neuronal responses to ITDs were quantified by circular statistical methods (Goldberg and Brown, 1969). The preferred interaural phase difference of a neuron is represented by the direction of the mean vector

The mean impulse number $\left(R_{\mathrm{i}}\right)$ at certain interaural phase difference $\beta$ defines a vector with two components $\left(x_{\mathrm{i}}\right.$ and $\left.y_{\mathrm{i}}\right)$. Multiplication of a MIP by the period of the stimulating frequency and division by $2 \pi$ gives rise to an interaural time difference.

Similarly, the degree of synchrony of impulses with the phase of the stimulus tone can be represented by the length of the mean vector, termed vector strength (VS), which is given by:

$$
\mathrm{VS}=\sqrt{\left(\left(\sum x_{\mathrm{i}}\right)^{2}+\left(\sum y_{\mathrm{i}}\right)^{2}\right) / \Sigma R_{\mathrm{i}}}
$$

where $R_{\mathrm{i}}$ is the mean impulse number at a certain phase angle and defines a vector with cosine and sine components $\left(x_{\mathrm{i}}\right.$ and $\left.y_{\mathrm{i}}\right)$, respectively, and $\Sigma R_{\mathrm{i}}$ is the sum of the mean impulse numbers for the entire period. VS varies from 0 , indicating no phase locking, to 1.0 , indicating all impulses occurring in one phase bin.

Summation ratio. The relationship between binaural and monaural responses was represented by the summation ratio (SR) (Goldberg and Brown, 1969):

$$
\mathrm{SR}=R_{\mathrm{b}}-R_{\text {spont }} /\left(\left(R_{\mathrm{i}}-R_{\text {spont }}\right)+\left(R_{\mathrm{c}}-R_{\text {spont }}\right)\right),
$$

where $R_{\mathrm{b}}$ is the response to binaural stimulation, $R_{\mathrm{i}}$ and $R_{\mathrm{c}}$ are the responses to monaural stimulation at the ipsilateral and the contralateral ears, respectively, and $R_{\text {spont }}$ is the spontaneous discharge. A value of 1 indicates linear summation, whereas values less than 1 and greater than 1 indicate "facilitation" and "disfacilitation," respectively (Goldberg and Brown, 1969).

\section{Anatomy}

The positions of recording electrodes were marked with Neurobiotin $(2 \%$ in patch solution) in the last recording sessions in some of the animals. After tracer injection, the owls were overdosed with sodium pentobarbital (Nembutal, Abbott Laboratories) and perfused first with $0.9 \%$ saline in $0.1 \mathrm{M}$ phosphate butter, $\mathrm{pH} 7.4$, and second with $4 \%$ paraformaldehyde in $0.1 \mathrm{~m}$ phosphate buffer. Brains were blocked in the plane of electrode penetration, removed from the skull, and placed in $30 \%$ sucrose until they sank. They were then cut into $30 \mu \mathrm{m}$ sections with a freezing microtome, rinsed repeatedly in buffer, prebleached with $0.5 \% \mathrm{H}_{2} \mathrm{O}_{2}$ in phosphate 
Table 1. Quantitative data on discharge rate, dynamic range, and threshold

\begin{tabular}{llllr} 
& $N$ & $\begin{array}{l}\text { Maximal response } \\
(\text { impulses/sec })\end{array}$ & $\begin{array}{l}\text { Dynamic range } \\
(\mathrm{dB})\end{array}$ & \multicolumn{1}{c}{$\begin{array}{l}\text { Threshold } \\
(\mathrm{dB} \text { SPL })\end{array}$} \\
\hline $\mathrm{NM}$ & 21 & $423 \pm 113$ & $31.1 \pm 13.1$ & $6.5 \pm 12.5$ \\
$\mathrm{NL}_{\text {fav }}$ & 29 & $354 \pm 168$ & $21.0 \pm 12.5$ & $16.3 \pm 11.0$ \\
$\mathrm{NL}_{\text {unf }}$ & 23 & $180 \pm 101$ & $26.3 \pm 11.8$ & $18.6 \pm 17.2$ \\
$\mathrm{NL}_{\text {ipsi }}$ & 33 & $210 \pm 129$ & $26.8 \pm 19.6$ & $24.7 \pm 18.6$ \\
$\mathrm{NL}_{\text {contra }}$ & 36 & $242 \pm 125$ & $24.4 \pm 14.9$ & $26.3 \pm 18.9$
\end{tabular}

All values are means \pm SD. NM denotes both ipsilateral and contralateral NM axons, $\mathrm{NL}_{\mathrm{fav}}$ and $\mathrm{NL}_{\mathrm{unf}}$ stand for the responses of NL neurons to the most and least favorable ITDs, respectively, and $\mathrm{NL}_{\mathrm{ipsi}}$ and $\mathrm{NL}_{\text {contra }}$ stand for the responses of $\mathrm{NL}$ neurons to ipsilateral and contralateral monaural stimulation, respectively. $N$ is the sample size.

buffer for $10 \mathrm{~min}$, and incubated in buffered $\mathrm{ABC}$ reagent (Vector Laboratories, Burlingame, CA). After multiple rinses, the sections were reacted in a buffered solution containing $0.1 \%$ diaminobenzidine (Sigma, St. Louis, MO) and $0.01 \%$ nickel ammonium sulfate for $15 \mathrm{~min}$ and then incubated with $3 \% \mathrm{H}_{2} \mathrm{O}_{2}$ for 5-15 min. Sections were mounted onto gelatin-coated slides and counterstained with cresyl violet.

\section{RESULTS}

Data were obtained from a total of 49 neurons of which different groups were used for measurement of different sets of response properties. The number of neurons used for each set of experiments is mentioned in separate sections below.

\section{Rate-intensity curves}

Neuronal responses were recorded for average binaural intensities (ABIs) ranging from 0 to $80 \mathrm{~dB}$ SPL at the best frequencies (BFs) of the individual neurons. Rate-intensity curves were obtained for the following four stimulus conditions: (1) binaural stimulation with the most favorable ITD; (2) binaural stimulation with the least favorable ITD; (3) ipsilateral stimulation; and (4) contralateral stimulation. Rate-intensity curves were obtained for all four stimulus conditions in 24 neurons. The binaural stimulation with the most favorable ITD always yielded the highest impulse rate (Fig. $3 A-C$, Table 1). Twelve neurons $(50.0 \%)$ showed higher impulse rates to contralateral stimulation than they did to ipsilateral stimulation (Fig. $3 A, B$ ). Five neurons (20.8\%), however, showed opposite responses, i.e., the ipsilateral stimulation gave rise to a higher impulse rate than did the contralateral stimulation (Fig. 3C). The remaining 7 neurons $(29.1 \%$ ) did not show a preference for either side.

In 11 neurons (45.8\%; e.g., Fig. $3 A$ ), binaural stimulation with the least favorable ITD gave rise to lower impulse rates than monaural stimulation to either side. In 5 neurons (20.8\%; e.g., Fig. $3 B, C$ ), discharge rates to binaural stimulation with the least favorable ITD were lower than the higher of the monaural rates. In 8 neurons (33.3\%), binaural stimulation with the least favorable ITD elicited impulse rates comparable to those elicited by monaural stimuli. In two cases, the impulse rate for the least favorable ITD fell below the spontaneous activity level. All discharge rates increased monotonically with sound intensity and reached an asymptote at 40-50 dB SPL. The relationships between the four rate-intensity curves of a neuron tended to persist in the suprathreshold range of sound intensity. Some of these relationships are analyzed further below.

\section{Vector strength}

Rate-intensity curves show only how discharge rate changes with sound intensity. We examined whether the degree of phase locking during the presentation of each test ITD varied with sound intensity in the same neurons for which we obtained rate-intensity curves. Because vector strength varies with frequency, we did not attempt to lump data from different neurons for statistical verification of observations on single neurons. The degree of phase locking as represented by vector strength appeared to change in parallel with discharge rates (Fig. $3 D-F$ ). Vector strengths began to increase and reached an asymptote at lower intensities than discharge rates did, as similar findings were reported in previous studies (Johnson, 1980; Sullivan and Konishi, 1984). Further increases in sound intensity did not appear to have any systematic effects on vector strength. Vector strength-intensity curves for the most favorable ITD and monaural responses tended to follow the patterns shown by the rate-intensity curves. Thus, the vector strength for the most favorable ITD was always greater than that for any other stimulus condition. The vector strength for the least favorable ITD was too small and variable to be useful for quantitative comparison. It should be pointed out, however, that these parallel changes of vector strengths and discharge rates are not attributable to the dependence of the former on the latter but, rather, to the fact that both increase monotonically with sound intensity before they reach an asymptote beyond which they remain unchanged. We examined this independence by calculating vector strength with different numbers of impulses obtained from the same neurons for the same sound intensity. The results showed that vector strength did not vary with the number of impulses used (data not shown).

\section{Summation ratio}

The intensity tolerance of ITD processing is also seen in the relationship between binaural and monaural responses. The discharge rate for the most favorable ITD was always greater than the sum of monaural discharge rates. Conversely, the discharge rate for the least favorable ITD was less than either or both of the monaural rates. These relationships did not appear to change with sound intensity once this exceeded $30 \mathrm{~dB}$ SPL. Because the summation ratio quantifies these relationships, we obtained this ratio for different sound intensities. We calculated the mean SR for the most favorable and the least favorable ITDs and plotted SR against stimulus intensity (Fig. 4). For a wide range of intensities, SR values were larger than 1.0 for stimulation with the most favorable ITD (mean value $1.51 \pm 0.27$ for suprathreshold stimulation) and remained below 1.0 for stimulation with the least favorable ITD (mean value $0.50 \pm 0.05$ ).

\section{Mean interaural phase}

The effects of variations in ABI on ITD curves were studied in 19 NL neurons. The neurons were stimulated with their individual best frequencies, and ITD tuning curves were obtained for different ABIs. When these curves were overlaid as in Figure $5 A$, the peaks and troughs lined up, indicating that the mean interaural phase angles of the ITD curves were unaffected by changes in ABI. Stimulation at a subthreshold level (0 dB SPL) showed no ITD tuning, and stimulation at threshold ( $15 \mathrm{~dB}$ SPL) elicited minimal ITD-dependent modulation of discharge (Fig. $5 A$ ). The depth of modulation increased over the dynamic range $(10-40 \mathrm{~dB}$ SPL) and leveled off for intensities greater than $40 \mathrm{~dB}$ SPL. When the ITD curve for $50 \mathrm{~dB}$ in Figure $5 A$ was plotted against the curves for other ABI values, a family of straight lines resulted (Fig. 5B); their linearity indicates that discharge rates for all ITDs increased proportionally as ABI was augmented.

To examine whether these shifts in ITD curves involve changes in neuronal selectivity for ITD, we calculated mean interaural phases (MIPs) for ITD responses of 19 neurons and plotted 



Figure 3. Monaural and binaural rate-intensity curves. $A$, Example of an NL neuron that displayed a higher impulse rate to contralateral stimulation than to ipsilateral stimulation. Its impulse rate to binaural stimulation with the least favorable ITD was lower than either monaural impulse rate. $B$, Example of an NL neuron whose impulse rate for the least favorable ITD was higher than that for monaural, ipsilateral stimulation. $C$, Example of an NL neuron that showed a higher impulse rate for ipsilateral than for contralateral stimulation. Error bars indicate SEM. $D-F$, Changes in vector strength with sound intensity for binaural and monaural responses of neurons in $A-C$, respectively. 


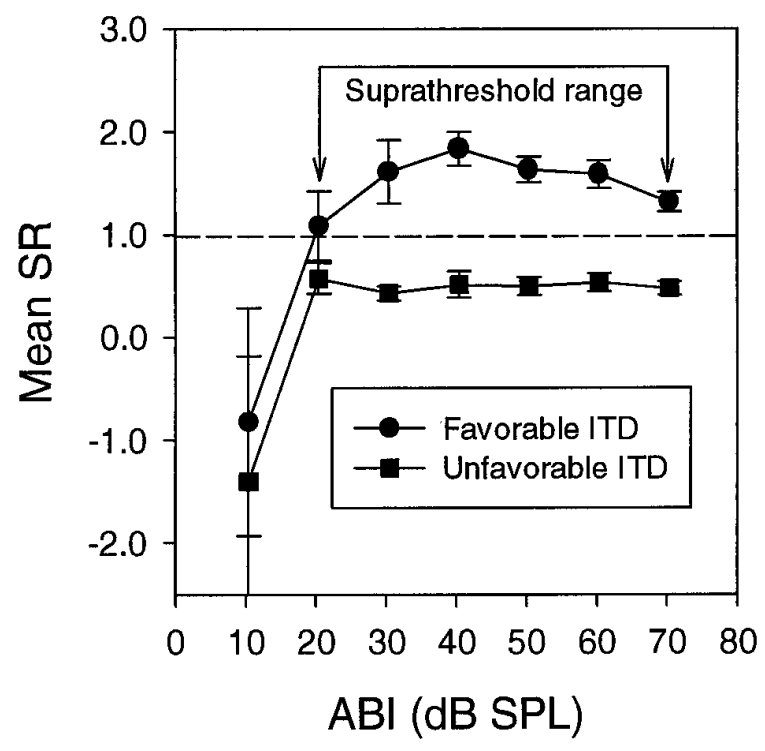

Figure 4. Relationship between summation ratio and average binaural intensity. Mean summation ratios of 24 NL neurons plotted against changes in average binaural intensity for most favorable (circles) and least favorable (squares) ITDs. The arrows indicate the suprathreshold range.

against ABI (Fig. 6). The results indicated that the MIP remained constant over a range of $\sim 60 \mathrm{~dB}$.

\section{Responses of afferent axons to variation in sound intensity}

The purpose of recording from NM fibers was to obtain evidence for or against the possibility that the intensity tolerance of NL neurons is attributable to the saturation of their afferent neurons. NM neurons could be distinguished easily from NL neurons, because NM neurons respond only to monaural stimulation. Furthermore, for the same stimulus intensity the mean first-impulse latency of NM fibers $(3.0 \pm 0.64 \mathrm{msec}, n=20)$ differed from that of monaural responses of NL neurons $(4 \pm 0.7 \mathrm{msec}, n=33$; $p<$ $10^{-4}, t$ test). Because the NM fibers were recorded inside NL, the difference of $1 \mathrm{msec}$ is consistent with the pre- and postsynaptic positions of NM and NL neurons, respectively. Both ipsilateral and contralateral fibers were encountered in all penetrations across NL as described previously by Carr and Konishi (1990). We obtained rate-intensity curves for $21 \mathrm{NM}$ fibers at their best frequencies. Figure 7 shows two examples of rate-intensity curves. Quantitative data on maximal response, dynamic range, and threshold obtained for the 21 neurons are summarized in Table 1.

\section{DISCUSSION}

All of the measured characteristics of NL neurons remained unchanged when sound intensity rose far above the level at which all impulse rates (for monaural and the most and least favorable ITDs) reached an asymptote. Goldberg and Brown (1969) also reported the insensitivity of ITD selectivity to variations in ABI in the dog's medial superior olivary nucleus, although they did not present quantitative data. Sound intensities between 40 and $50 \mathrm{~dB}$ SPL caused most NL neurons to reach an asymptotic firing level. However, the neurons continued to show ITD sensitivity far beyond the beginning of this "saturation" level. The asymptotic firing levels of NL neurons may be determined by those of their afferent fibers, which also reach asymptotes at sound intensities of 40-50 dB SPL. If a single NL neuron received input by one axon from each side, the saturation of the afferent fiber would suffice to account for that of the postsynaptic


Figure 5. Effect of intensity on the mean interaural phase. A, Overlaid ITD curves for different average binaural intensities, indicated on the right in $\mathrm{dB}$ SPL. The peaks and troughs line up, indicating that the MIP was independent of ABI. $B$, Linear changes in ITD curves with average binaural intensity. The ITD curve for $50 \mathrm{~dB}$ (reference response) is plotted against other ITD curves in $A$.

neuron. A single NL neuron may receive, however, as many as 45-150 afferent fibers from each side (Carr and Boudreau, 1993).

The convergence of many afferent fibers on a single NL neuron would seem useful for increasing the probability of binaural coincidence, especially for high frequencies. However, this condition also increases the probability of monaural coincidence that results from simultaneous arrival of impulses conveyed by fibers coming from the same side. Threshold crossing caused by monaural coincidences may become as frequent as that caused by binaural coincidences as the afferent impulse rate increases. This condition would lead to flattening of ITD curves, because the coincidence detector can fire independently of ITD. Because there are twice as many fibers for binaural coincidence as for monaural coincidence, the probability of binaural coincidence would be greater than that of monaural coin- 




Figure 6. The immunity of mean interaural phase to changes in average binaural intensity. Neurons $(n=19)$ were stimulated with tone bursts at their best frequencies. Plots of MIP against ABI are horizontal lines indicating that MIP remained largely unchanged over a $70 \mathrm{~dB}$ range.

cidence. Therefore, if the threshold is appropriately adjusted, binaural coincidences trigger more impulses than monaural coincidences. Inhibitory input that varies the detector's threshold of discharge with sound intensity is one way to prevent indiscriminate threshold crossing, although control of threshold without inhibitory input cannot be excluded. The anatomical substrates for inhibitory control of NL exist. This nucleus is heavily innervated by GABAergic fibers in both chickens and barn owls (Carr et al., 1989; Lachica et al., 1994). When hodological and neurochemical data from chickens and barn owls are compared and combined, the following relationships between the brainstem auditory nuclei can be found. The superior olivary nucleus (SO) receives excitatory input from nucleus angularis (NA) and NL and sends GABAergic fibers to these nuclei and to NM (Takahashi and Konishi, 1988b; Carr et al., 1989; Carr and Boudreau, 1993; Lachica et al., 1994). Lachica et al. (1994) suggested that these GABAergic fibers control the gain of neurons in the target nuclei.

To explore the role of inhibition in providing immunity against high-intensity sounds, we built a model similar to the one described by Colburn et al. (1990). The only difference is that our coincidence detectors receive both excitatory and inhibitory input (Fig. $8 A$ ). In our model, NL is represented by one neuron that receives two sets of five excitatory inputs and two sets of five inhibitory inputs. One set of excitatory inputs comes from five model neurons in the ipsilateral $\mathrm{NM}$, and the other comes from the contralateral NM. The two sets of inhibitory inputs come from the ipsilateral and contralateral SO. Each of these neurons is innervated by one NA neuron. Five NA neurons represent the ipsilateral NA, and the other five the contralateral NA. The SO inhibitory neurons respond every time the NA neurons that innervates them fire. The NM neurons are similar to the input fibers of Colburn et al. (1990). Their firing probability density is given by:

$$
P(t)=a_{0} \exp \left(a_{1} \cos (2 \pi f t+\theta)\right) \mathrm{d} t,
$$

where $\mathrm{d} t$ is the time bin, set to $100 \mu$ sec. $a_{0}$ in this expression represents the intensity, and $a_{1}$ controls the degree of phase locking. Each of these neurons has a refractory period of $1.0 \mathrm{msec}$. Following Colburn et al. (1990), we set $a_{1}=2.7$.
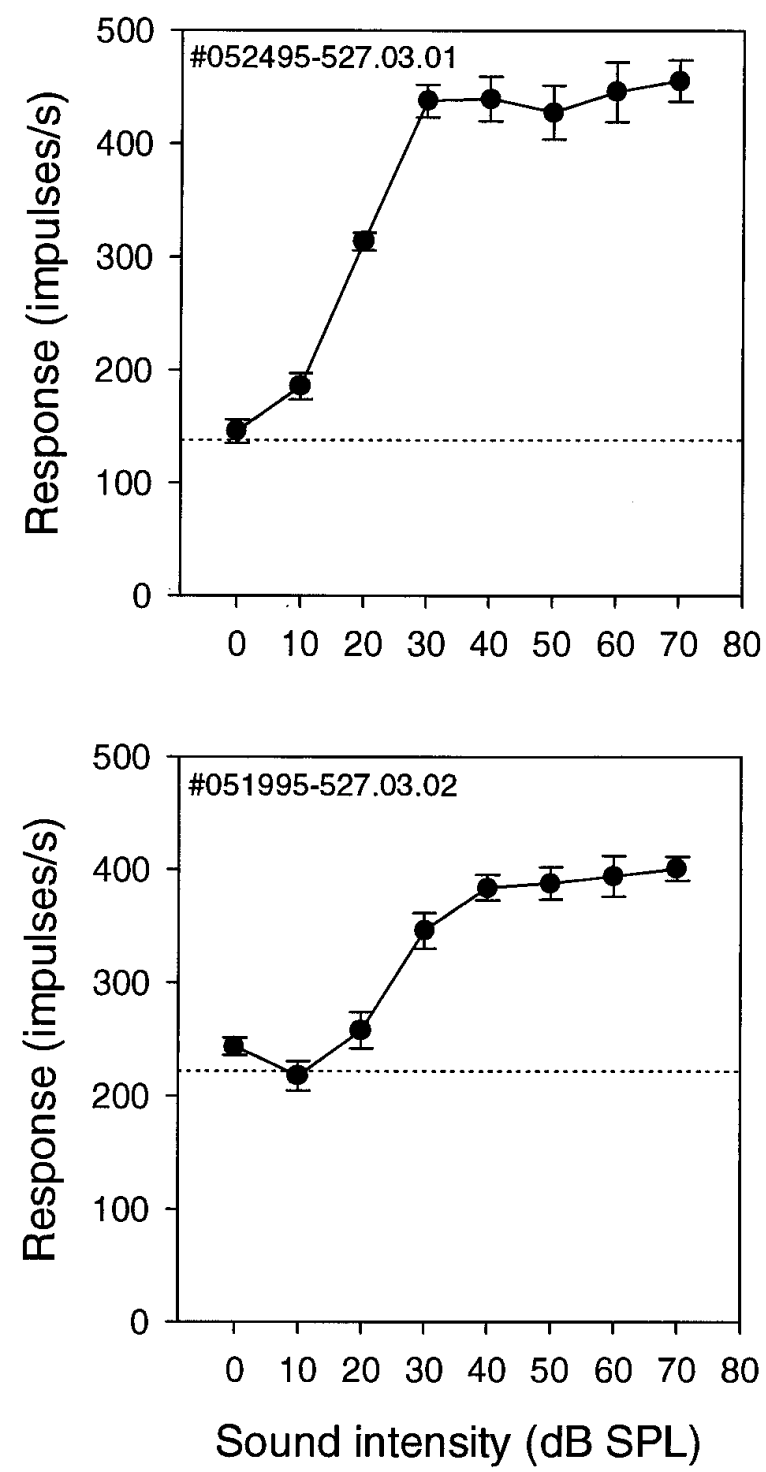

Figure 7. Rate-intensity curves of two fibers from nucleus magnocellularis. Both spontaneous (shown by dotted line) and asymptotic discharge rates are higher in NM cells than in NL cells, whereas the dynamic range $(\sim 30 \mathrm{~dB})$ is similar in the two nuclei (compare Table 1$)$.

The NA neurons do not phase-lock (i.e., $a_{1}=0$ ) and have a firing rate comparable to that of NM neurons. Also, they saturate at higher sound intensities. We assume that the SO inhibitory neurons are driven by NA, and the degree of inhibition is supposed to be proportional to that of excitation in the NA neurons. Figure $8 B$ shows the rate-intensity curves of NM and NA neurons in terms of firing rate versus the parameter $a_{0}$.

NL neurons are modeled with two hypothetical synaptic currents. The excitatory synaptic current increases by one unit each time an impulse from one of the NM neurons arrives. Then the current decays exponentially with time constant $\tau_{\mathrm{ex}}=1 \mathrm{msec}$. The second current, the inhibitory one, increases with each impulse from SO and, similarly, it decays with a time constant of $\tau_{\text {in }}=2 \mathrm{msec}$. The amount of increase in response to the SO impulse determines the weight of the inhibitory effect. The NL neuron fires an impulse every time the difference between the currents exceeds a threshold of 3 units. The refractory period of the NL neuron is $1 \mathrm{msec}$.

When the inhibitory weight is set to zero, the model resembles 

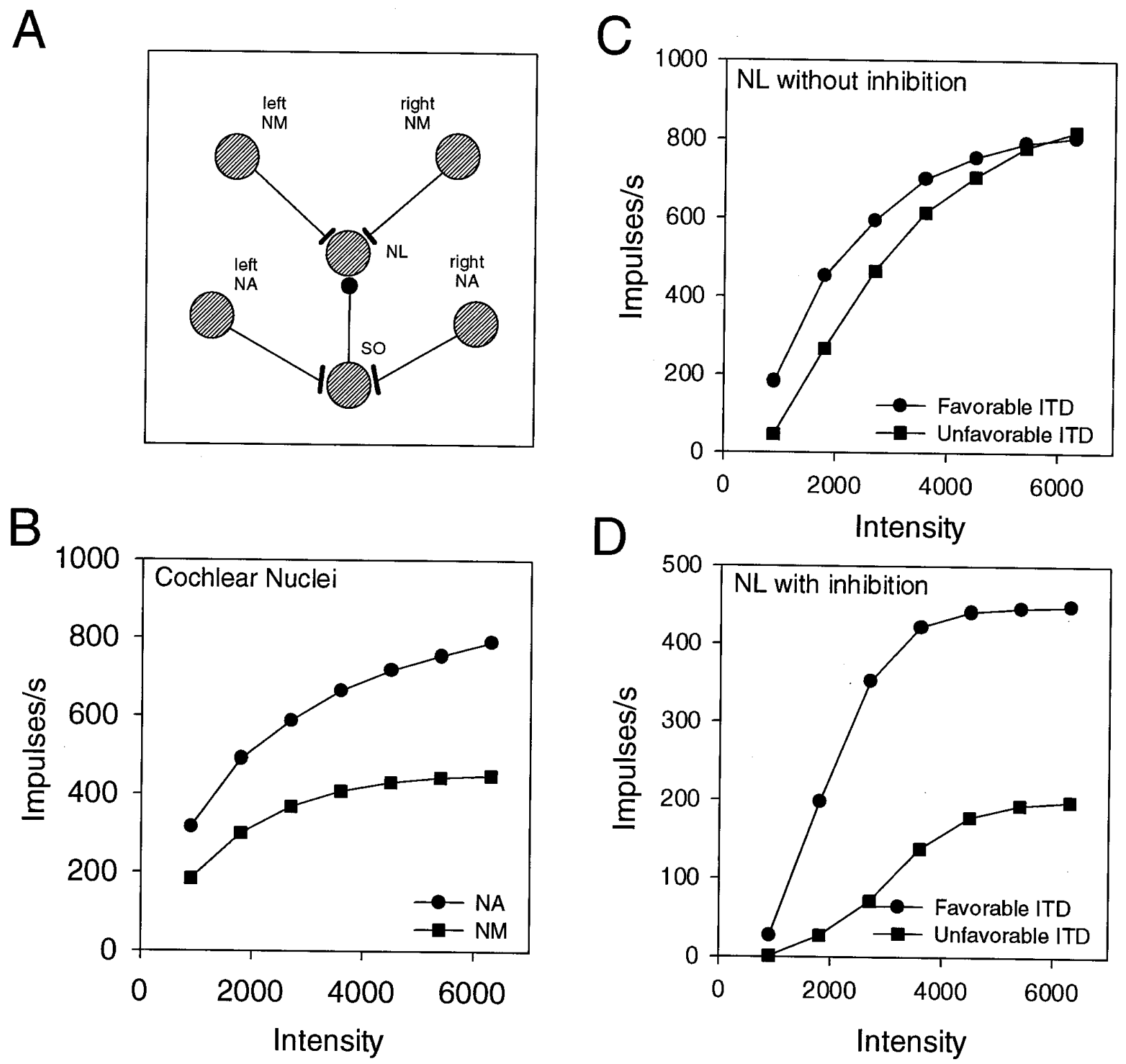

Figure 8. Effect of inhibitory input to a nucleus laminaris neuron: a model. $A$, Excitatory and inhibitory inputs to a nucleus laminaris neuron. The inhibitory input is assumed to originate from SO. NA neurons are assumed to drive SO-inhibitory neurons. $B$, Rate-intensity curves of NA and NM neurons. Sound intensities on the abscissa are in arbitrary units ( $a_{0}$ in the equation). $C$, Intensity dependence of a model without inhibition. As in Colburn et al. (1990), the model fits the data of Goldberg and Brown (1969) when $a_{0}=900$. As intensity increases (input fibers approach a mean firing rate of $\sim 400$ impulses/sec), the curves for the most and least favorable ITDs merge, indicating that the neuron loses its ITD selectivity. $D$, The effect of inhibition on the same model. Here, the neuron maintains a constant ratio between the responses to the most and least favorable ITDs.

the one described by Colburn et al. (1990). The effect of intensity on this model without inhibition is described in Figure $8 C$. For moderate sound intensities $\left(a_{0}<3500\right)$, the response to the most and least favorable ITDs increase at the same rate. This means that the ratio between them decreases. At higher intensities, the response to the least favorable ITD becomes comparable to the response to the most favorable ITD and even exceeds it at higher levels. Figure $8 D$ describes the effect of intensity on the model when inhibition weight of 0.2 is used. Under this condition, the responses of the model NL neuron to both the most and the least favorable ITDs increase in proportion to sound intensity such that the ratio between them remains relatively constant.

The problem of sound intensity in coincidence detection has also been addressed in another modeling study (Reed and Durbeck,
1995). The authors suggested two types of inhibition for the solution of this problem. One requires accurate timing of excitatory and inhibitory inputs, a possibility that we did not investigate. The other is similar to our model except that their model uses inhibition that sets in when intensity increases beyond a certain level. The authors predicted that this mechanism would provide NL neurons with a dynamic range that is greater than that of NM neurons. This prediction is contradicted by our results, in which we observed similar dynamic ranges in NL and NM. In our model, slight differences in the rate-intensity relation between NM and NA can maintain NL neurons within their operational range without increasing their dynamic range. The same effect can be achieved by inhibitory feedback. A candidate would be an excitatory connection from NL to SO and a reciprocal inhibitory connection from SO to NL. 
The possible involvement of inhibition in the processing of ITD has been pointed out in several studies of neurons of the cat's inferior colliculus and MSO. Binaural discharge rates drop below either or both monaural levels and, in some neurons, even below the spontaneous level. When these phenomena occur in the inferior colliculus, they may indicate inhibition (Rose et al., 1966; Yin and Kuwada, 1983; Carney and Yin, 1989). However, the same phenomena in the MSO are thought by some authors to involve inhibition (Yin and Kuwada, 1984; Yin and Chan, 1988, 1990; Spitzer and Semple, 1995) and not by other authors (Colburn et al., 1990; Reyes et al., 1996). Goldberg and Brown (1969) coined the term "disfacilitation" to describe the discharge rate for the least favorable ITD being lower than the monaural rates. The physiological proof for the presence of inhibitory input to coincidence detectors must come from intracellular recordings and neuropharmacological experiments. Grothe and Sanes (1993, 1994) showed in brain slices that MSO neurons of gerbils receive inhibitory input the magnitude of which varies with stimulus voltage. Hyson et al. (1995) described in the chick's nucleus magnocellularis and nucleus laminaris a unique form of GABA receptor that inhibits by depolarization instead of hyperpolarization. The authors suggested that this form of GABA receptor might be efficient in reducing large EPSPs. $\mathrm{GABA}_{\mathrm{A}}$ receptors that are blocked by bicucculine are also present in the chick's nucleus laminaris. The role of these receptors is thought to shorten the duration of EPSPs (Funabiki et al., 1995).

Despite these findings, there is no direct evidence that $\mathrm{GABA}_{\mathrm{A}^{-}}$ mediated inhibition contributes to the shape of ITD curves in vivo. Also, direct evidence for the gain control function of inhibition in the owl's nucleus laminaris is lacking. In the present paper, we show that the owl's NL neurons do not lose their selectivity for interaural time difference with intense sound and conclude that this tolerance to intensity is inconsistent with models of binaural coincidence detection without inhibitory input.

\section{REFERENCES}

Albeck Y (1994) A model of inhibition as a gain control in the early processing of interaural time difference in the barn owl's brainstem. Soc Neurosci Abstr 20:136.

Caird D, Klinke R (1983) Processing of binaural stimuli by cat superior olivary complex neurons. Exp Brain Res 52:385-399.

Carney LH, Yin TCT (1989) Responses of low-frequency cells in the inferior colliculus to interaural time differences of clicks: excitatory and inhibitory components. J Neurophysiol 62:144-161.

Carr CE, Boudreau RE (1993) Organization of the nucleus magnocellularis and the nucleus laminaris in the barn owl: encoding and measuring interaural time differences. J Comp Neurol 334:337-355.

Carr CE, Konishi M (1988) Axonal delay lines for time measurement in the owl's brainstem. Proc Natl Acad Sci USA 85:8311-8315.

Carr CE, Konishi M (1990) A circuit for detection of interaural time differences in the brain stem of the barn owl. J Neurosci 10:3227-3246.

Carr CE, Fujita I, Konishi M (1989) Distribution of GABAergic neurons and terminals in the auditory system of the barn owl. J Comp Neurol 286:190-207.

Colburn HS, Han Y, Culotta CP (1990) Coincidence model of MSO responses. Hear Res 49:335-346.

Crow G, Rupert AL, Moushegian G (1978) Phase locking in monaural and binaural medullary neurons: implications for binaural phenomena. J Acoust Soc Am 64:493-501.

Funabiki K, Koyano K, Ohmori H (1995) Postsynaptic responses of the nucleus laminaris of the chick. Soc Neurosci Abstr 21:128.

Goldberg JM, Brown PB (1969) Response of binaural neurons of dog superior olivary complex to dichotic tonal stimuli: some physiological mechanisms of sound localization. J Neurophysiol 32:613-636.

Grothe B, Sanes DH (1993) Bilateral inhibition by glycinergic afferents in the medial superior olive. J Neurophysiol 69:1192-1195.
Grothe B, Sanes DH (1994) Synaptic inhibition influences the temporal coding properties of medial superior olivary neurons: an in vitro study. J Neurosci 14:1701-1709.

Guinan Jr JJ, Guinan SS, Norris BE (1972) Single auditory units in the superior olivary complex. I. Responses to sounds and classifications based on physiological properties. Int J Neurosci 4:101-120.

Hopfield JJ (1995) Pattern-recognition computation using actionpotential timing for stimulus representation. Nature 376:33-36.

Hyson RL, Reyes AD, Rubel EW (1995) A depolarizing inhibitory response to GABA in brain-stem auditory neurons of the chick. Brain Res 677:117-126.

Jeffress LA (1948) A place theory of sound localization. J Comp Physiol Psychol 41:35-39.

Johnson DH (1980) The relationship between spike rate and synchrony in response of auditory-nerve fibers to single tones. J Acoust Soc Am 68:1115-1122.

Lachica EA, Rübsamen R, Rubel EW (1994) GABAergic terminals in nucleus magnocellularis and laminaris originate from the superior olivary nucleus. J Comp Neurol 348:403-418.

Mazer JA (1995) Integration of parallel processing streams in the inferior colliculus of the barn owl. $\mathrm{PhD}$ thesis, California Institute of Technology.

Moiseff A, Konishi M (1981) The owl's interaural pathway is not involved in sound localization. J Comp Physiol [A] 144:299-304.

Moiseff A, Konishi M (1983) Binaural characteristics of units in the owl's brainstem auditory pathway: precursors of restricted spatial receptive fields. J Neurosci 3:2553-2562.

Moushegian G, Rupert A, Whitecomb MA (1964) Medial superiorolivary-unit response patterns to monaural and binaural clicks. J Acoust Soc Am 36:196-202.

Moushegian G, Rupert AL, Langford TL (1967) Stimulus coding by medial superior olivary neurons. J Neurophysiol 30:1239-1261.

Moushegian G, Rupert AL, Gidda JS (1975) Functional characteristics of superior olivary neurons to binaural stimuli. J Neurophysiol 38:1037-1048.

Reichardt W (1961) Autocorrelation, a principle for the evaluation of sensory information by the central nervous system. In: Sensory communication (Rosenblith WA, ed), pp 303-317. Cambridge: MIT.

Reed CR, Durbeck L (1995) Delay lines and auditory processing. In: Comments on modern biology, Vol C, Comments on theoretical biology, pp 441-461. London: Gordon and Breach.

Reyes AD, Rubel EW, Spain WJ (1996) In vitro analysis of optimal stimuli for phase-locking and time-delayed modulation of firing in avian nucleus laminaris neurons. J Neurosci 16:993-1007.

Rose JE, Gross NB, Geisler CD, Hind JE (1966) Some neural mechanisms in the inferior colliculus of the cat which may be relevant to localization of a sound source. J Neurophysiol 29:288-314.

Rupert A, Moushegian G, Whitcomb MA (1966) Superior-olivary response patterns to monaural and binaural clicks. J Acoust Soc Am 39:1069-1076.

Spitzer MW, Semple MN (1995) Neurons sensitive to interaural phase disparity in gerbil superior olive: diverse monaural and temporal response properties. J Neurophysiol 73:1668-1690.

Suga N, Olsen JF, Butman JA (1990) Specialized subsystems for processing biologically important complex sounds: cross-correlation analysis for ranging in the bat's brain. Cold Spring Harbor Symp Quant Biol 55:585-595.

Sullivan WE, Konishi M (1984) Neural map of interaural phase difference in the owl's brainstem. Proc Natl Acad Sci USA 83:8400-8404.

Takahashi T, Konishi M (1988a) Projections of the cochlear nucleus and the nucleus laminaris to the inferior colliculus of the barn owl. J Comp Neurol 274:190-211.

Takahashi T, Konishi M (1988b) Projections of nucleus angularis and nucleus laminaris to the lateral lemniscal nuclear complex of the barn owl. J Comp Neurol 274:212-238.

Yin TCT, Chan JCK (1988) Neural mechanisms underlying interaural time sensitivity to tones and noise. In: Auditory function (Edelman GM, Gall WE, Cowan WM, eds), pp 385-430. New York: Wiley.

Yin TCT, Chan JCK (1990) Interaural time sensitivity in the medial superior olive of the cat. J Neurophysiol 64:465-488.

Yin TCT, Kuwada S (1983) Binaural interaction in low-frequency neurons in inferior colliculus of the cat. II. Effects of changing rate and direction of interaural phase. J Neurophysiol 50:1000-1019.

Yin TCT, Kuwada S (1984) Neuronal mechanisms of binaural interaction. In: Dynamic aspects of neocortical function (Edelman GM, Gall WE, Cowan WM, eds), pp 263-313. New York: Wiley. 